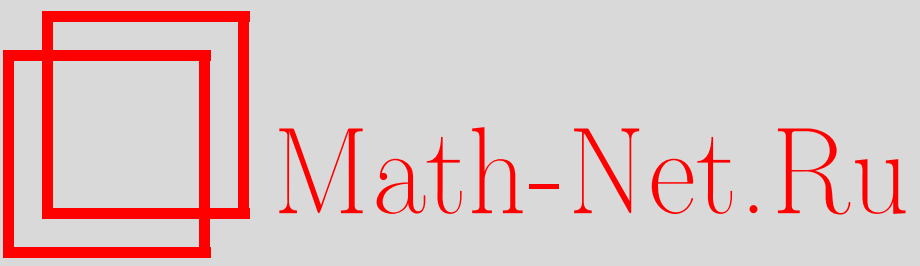

В. П. Маслов, Проблема фазовых переходов в сверхтекучей и нормальной жидкостях, ТМФ, 1999, том 121, номер 3, 492-508

DOI: https://doi.org/10.4213/tmf825

Использование Общероссийского математического портала Math-Net.Ru подразумевает, что вы прочитали и согласны с пользовательским соглашением

http://www.mathnet.ru/rus/agreement

Параметры загрузки:

IP : 54.147 .182 .235

26 апреля 2023 г., 04:03:28 


\section{ПРОБЛЕМА ФАЗОВЫХ ПЕРЕХОДОВ В СВЕРХТЕКУЧЕЙ И НОРМАЛЬНОЙ ЖИДКОСТЯХ ${ }^{1)}$}

Излагается новая точка зрения на известный спектр, полученный Боголюбовым для бозонного газа, и на роль угловой скорости как термодинамической величины, определяющей фазовый переход второго рода. Предъявляется новый способ осреднения по кластерам, приводящий к новой спектральной серии и другому фазовому переходу. Этот способ обобщается на классические уравнения, что позволяет высказать гипотезу о фазовом переходе по скорости ламинарного потока в турбулентный.

Угловая скорость является термодинамической переменной. Рассмотрение задач на торе, как это обычно делается (например, Боголюбовым), демонстрирует, что решение задачи, инвариантной относительно сдвигов вдоль тора, имеет параметром угловую скорость. Из работы Боголюбова о слабонеидеальном бозе-газе (1947 г.) следует, что по угловой скорости совершается фазовый переход из сверхтекучего состояния в нормальное. В настоящей статье изучаются фазовые переходы для случая, когда бозоны осредняются по парам и более сложным кластерам. Отметим, что этот метод осреднения в случае фермионов приводит к уравнениям Бардина-Купера-Шрифера-Боголюбова.

В классической механике для многочастичных уравнений Лиувилля также имеют место фазовые переходы. По-видимому, один из переходов можно трактовать как переход в турбулентное течение, а другой (для газа) - как переход от дозвукового в сверхзвуковое течение.

1. Вначале в качестве примера мы рассмотрим схематично задачу о слабонеидеальном бозе-газе, решенную Боголюбовым с помошью знаменитых $u-v$-преобразований в 1947 г., с точки зрения асимптотических методов $[1,2]$. Эта точка зрения позволяет взглянуть на известные формулы с других позиций.

Уравнение Шредингера

$$
\begin{aligned}
i \hbar \frac{\partial \Psi}{\partial t}\left(x_{1}, \ldots, x_{N} ; t\right)= & -\frac{\hbar^{2}}{2 m} \sum_{j=1}^{N} \Delta_{j} \Psi\left(x_{1}, \ldots, x_{N} ; t\right)+ \\
& +U_{0} \sum_{1 \leqslant j<k \leqslant N} V\left(\frac{x_{j}-x_{k}}{a}\right) \Psi\left(x_{1}, \ldots, x_{N} ; t\right) \equiv \widehat{H}_{N} \Psi,
\end{aligned}
$$

\footnotetext{
1) Статья публикуется в дискуссионном порядке. Реджоллегия.
}

* Московский государственный университет, Москва, Россия 
где $\Psi\left(x_{1}, \ldots, x_{N} ; t\right) \in L^{2}\left(\mathbf{T}^{3 N}\right)$ - симметрическая функция аргументов $x_{i}, \Delta_{j}$ - оператор Лапласа, $V(x) \in C^{\infty}, V(x)=V(-x)$, решается асимптотически при $N \rightarrow \infty$, объеме $\mathcal{V} \rightarrow \infty, N / \mathcal{V} \rightarrow$ const при обычных условиях периодичности типа Борна-Кармана. В представлении вторичного квантования оно имеет вид

$$
i \hbar \frac{\partial \Phi(t)}{\partial t}=\widehat{H} \Phi(t)
$$

где оператор $\widehat{H}$ имеет вид

$$
\widehat{H}=\int d x \hat{b}^{+}(x)\left(-\frac{\hbar^{2}}{2 m} \Delta\right) \hat{b}^{-}(x)+\frac{U_{0}}{2} \iint d x d y V\left(\frac{x-y}{a}\right) \hat{b}^{+}(x) \hat{b}^{+}(y) \hat{b}^{-}(y) \hat{b}^{-}(x),
$$

здесь $\hat{b}^{ \pm}(x)$ - операторы рождения и уничтожения в бозонном пространстве Фока; $\Phi(t)$ - элементы пространства Фока.

В нашем приближении можно считать операторы рождения и уничтожения коммутируюшими ( $c$-числами). Действительно, после приведения к параметру $\epsilon=1 / N$ можно получить в известном представлении для $b^{+}$и $b^{-}$формулы

$$
b^{+}(x)=\epsilon \frac{\partial}{\partial q_{x}}+q_{x}, \quad b^{-}(x)=\epsilon \frac{\partial}{\partial q_{x}}-q_{x} .
$$

Это приведет к уравнению

$$
i \hbar \frac{\partial \varphi(x, t)}{\partial t}=-\frac{\hbar^{2}}{2 m} \Delta \varphi(x, t)+N U_{0} \int d y V\left(\frac{x-y}{a}\right)|\varphi(y, t)|^{2} \varphi(x, t),
$$

где $\varphi(x, t) \in L^{2}\left(\mathbf{T}^{3}\right)$. Эта ситуация при $U_{0} \sim 1 / N$ аналогична классическому пределу Гайзенберга, когда операторы $\hat{p}=i \hbar \partial / \partial x$ и $x$ становятся коммутативными ( $c$-числами).

Уравнение (3) является бесконечномерной гамильтоновой системой, у которой $\operatorname{Re} \varphi$ - бесконечномерный импульс, $a \operatorname{Im} \varphi$ - бесконечномерная координата. Периодические по $t$ решения этого уравнения являются, таким образом, одномерными замкнутыми траекториями в бесконечномерном фазовом пространстве, и к задаче (2) может быть применена теория комплексного ростка [2]. Это значит, что серии собственных значений для задачи (1) и отвечаюшие им собственные функции будут определяться с помошью спектра уравнений в вариациях к (3), которые имеют вид

$$
\begin{aligned}
-\beta F(x)= & \left(-\frac{\hbar^{2}}{2 m} \Delta-\Omega\right) F(x)+N U_{0} \int d y V\left(\frac{x-y}{a}\right)|\varphi(y)|^{2} F(x)+ \\
& +N U_{0} \int d y V\left(\frac{x-y}{a}\right)\left(G(y) \varphi(y)+\varphi^{*}(y) F(y)\right) \varphi(x), \\
\beta G(x)= & \left(-\frac{\hbar^{2}}{2 m} \Delta-\Omega\right) G(x)+N U_{0} \int d y V\left(\frac{x-y}{a}\right)|\varphi(y)|^{2} G(x)+ \\
& +N U_{0} \int d y V\left(\frac{x-y}{a}\right)\left(G(y) \varphi(y)+\varphi^{*}(y) F(y)\right) \varphi^{*},
\end{aligned}
$$


при условии, что в качестве периодического решения уравнения (3) взята функция $\varphi(x, t)=\varphi(x) e^{-i \frac{\Omega}{\hbar} t}$.

Частным решением уравнения (3) является

$$
\varphi(x)=\exp \left(\frac{i m v_{0} x}{\hbar}\right)
$$

c "собственным значением" $\Omega=$ const $+m v_{0}^{2} / 2$.

Спектр (4) “около” этого решения имеет вид известной формулы Боголюбова:

$$
\beta\left(k, v_{0}\right)=\left(\bar{k}, \bar{v}_{0}\right)+\sqrt{\tilde{V}(\bar{k}) \bar{k}^{2}+\frac{\hbar^{2} \bar{k}^{4}}{4}},
$$

где $\widetilde{V}(\bar{k})$ - преобразование Фурье потенциала взаимодействия $V(x)$.

Классическое приближение достаточно хорошее. Если в формуле (5) положить $\hbar=0$, то получится соотношение, которое может быть получено из классического уравнения в вариациях (см. [3]).

На основе (5) для уравнения (1) строится серия собственных значений вида

$$
\sum_{k} \beta\left(k, v_{0}\right) n_{k}+\text { const }
$$

где $n_{k}$ - любые целые положительные числа. Собственные функции, отвечающие этим собственным значениям, асимптотически выражаются через канонический оператор с комплексным ростком [2].

2. Отметим, что асимптотика собственных функций уравнения (1) не была получена ни в работе Боголюбова, ни в дальнейших разработках. Но именно она позволяет установить следуюшее важнейшее свойство: переходы с этой серии на другие уровни оператора $\widehat{H}_{N}$ в (1) “почти” запрешены - они экспоненциально малы по $1 / N$. Этим оправдывается то, что в физической литературе пишется распределение Бозе-Эйнштейна по "квазичастицам" (5). Действительно, для достаточно больших, но не бесконечно больших времен $t=t_{0}$ локальное тепловое равновесие может установиться по серии (5), параметризуемой вектором $v_{0}$, подобно тому как в $n$ сообшаюшихся сосудах, соединенных с другими $k$ сообшаюшимися сосудами тонким капилляром, равновесие жидкости устанавливается за время $t_{0}$, в то время как за “очень" большое время равновесное состояние будет другим - жидкость наполнит все $n+k$ сосудов одинаково. Мы можем, таким образом, по (6) построить гиббсовскую большую статистическую сумму $\sum$ для данной температуры $\Theta=k T$ ( $T$ - абсолютная температура, $k$ - константа Больцмана). Она и будет совпадать с распределением Бозе-Эйнштейна по квазичастицам. При этом вектор скорости $v_{0}$ будет играть роль новой (интенсивной) термодинамической величины, а импульс $\bar{p}_{0}=\partial \sum / \partial \bar{v}_{0}-$ сопряженной к ней (экстенсивной) термодинамической величины.

Поскольку оказьвается, что $\bar{p}_{0}=\rho\left(\bar{v}_{0}, \Theta\right) \bar{v}_{0}$, то $\rho\left(\bar{v}_{0}, \Theta\right)$ можно считать плотностью нормальной компоненты жидкости, увлекаемой движущимся капилляром, т.е. рассматривать двухжидкостную модель Ландау. При $\Theta=0$ и $v_{0}=v_{0_{\text {кр }}}$ (критической скорости 
Ландау) происходит фазовьй переход. Отметим, что для очень больших времен в силу сказанного выше равновесное распределение будет совсем другое.

Тот факт, что переходы с одной серии на другую “почти запрешены”, может быть доказан следующим образом. Нужно, как это делал Боголюбов, рассмотреть исходную задачу на торе радиуса $L$, тогда спектр уравнения (3) будет дискретным и, следовательно, серии будут нумероваться дискретным набором скоростей $v_{s}, s=0,1, \ldots$. Уравнение (3) и комплексно сопряженное ему являются с точки зрения указанного выше соответствия бесконечномерной системой Гамильтона, где $\operatorname{Re} \psi$ и $\operatorname{Im} \psi$ играют роль координат и импульсов в классической механике.

3. Эта точка зрения позволяет выписать асимптотику собственных функций задачи (1) при $N \rightarrow \infty, u_{0} \sim 1 / N$, причем не только с точностью до $(1 / N) k$, где $k$ любое, но и получить “экспоненциальные хвосты” этих собственных функций. Это последнее обстоятельство позволяет получить инстантоны, а также оценить указанные вьше матричные элементы.

После выделения циклической переменной - числа частиц $N$ - и перехода к мнимым импульсам можно получить туннельный гамильтониан следующего вида:

$$
\begin{aligned}
H(q, p)= & \sum_{k \neq 0} \frac{\hbar^{2}}{2 m L^{2}}(2 \pi k)^{2} \frac{1}{2}\left(q_{k}^{2}-p_{k}^{2}\right)+\frac{U_{0} a^{3}}{2 L^{3}} \tilde{v}(0)\left(N-\sum_{k \neq 0} \frac{1}{2}\left(q_{k}^{2}-p_{k}^{2}\right)\right)+ \\
& +\frac{U_{0} a^{3}}{2 L^{3}} \sum_{k \neq 0} \tilde{v}\left(\frac{2 \pi a}{L} k\right)\left(q_{k} q_{-k}+p_{k} p_{-k}\right)\left(N-\sum_{l \neq 0} \frac{1}{2}\left(q_{l}^{2}-p_{l}^{2}\right)\right)+ \\
& +\frac{U_{0} a^{3}}{L^{3}} \sum_{k \neq 0} \tilde{v}\left(\frac{2 \pi a}{L} k\right) \frac{1}{2}\left(q_{k}^{2}-p_{k}^{2}\right)\left(N-\sum_{l \neq 0} \frac{1}{2}\left(q_{l}^{2}-p_{l}^{2}\right)\right)+ \\
& +\frac{U_{0} a^{3}}{L^{3}} \sum_{k, l, m \neq 0} \tilde{v}\left(\frac{2 \pi a}{L} k\right) \Delta(k+l-m) \frac{1}{2 \sqrt{2}}\left(\left(q_{k}+p_{k}\right)\left(q_{l}+p_{l}\right)\left(q_{m}-p_{m}\right)+\right. \\
& \left.+\left(q_{m}+p_{m}\right)\left(q_{l}-p_{l}\right)\left(q_{k}-p_{k}\right)\right) \sqrt{N-\sum_{n \neq 0} \frac{1}{2}\left(q_{n}^{2}-p_{n}^{2}\right)+} \\
& +\frac{U_{0} a^{3}}{2 L^{3}} \sum_{k, l, m, n} \tilde{v}\left(\frac{2 \pi a}{L}(k-n)\right) \Delta(k+l-m-n) \times \\
& \times\left(q_{k}+p_{k}\right)\left(q_{l}+p_{l}\right)\left(q_{m}-p_{m}\right)\left(q_{n}-p_{n}\right),
\end{aligned}
$$

где $L$ - длина стороны трехмерного тора, $u_{0}$ и $а$ определяются из потенциала взаимодействия

$$
V(x, y)=U_{0} v\left(\frac{x-y}{a}\right),
$$

$\tilde{v}(q)$ - фурье-преобразование от $v(x)$,

$$
\tilde{v}(q)=\int d x v(x) e^{-i q x},
$$

$\Delta(k)=1$ при $k=0 ; \Delta(k)=0$ при $k \neq 0$. 
Асимптотика дается туннельным каноническим оператором, отвечающим этому гамильтониану. Отметим, что действие, вьгисленное вдоль “классической” траектории, отвечающей этому гамильтониану, возрастает вдоль “классического” пути.

4. Приведенное выше рассмотрение неполно в том смысле, что: 1) рассмотрено лиш частное решение уравнения (3), имеюшего, вообше говоря, другие периодические по времени решения, т.е. другие замкнутые траектории гамильтоновой системы, причем, возможно, “энергетически более выгодные” ; 2) уравнение (3) отвечает вариационному принципу, когда ищется решение уравнения (1) в виде

$$
\Psi=\varphi\left(x_{1}\right) \varphi\left(x_{2}\right) \ldots \varphi\left(x_{N}\right)
$$

Однако если потенциал взаимодействия не чисто отталкивающий, как это имело место в рассмотрении Боголюбова, а вблизи своего минимума притягивающий, как это имеет место в реальных взаимодействиях, то оказывается, что нашим состояниям отвечает такое уравнение Гамильтона, которое получается, если решение искать вариационным методом в более обшем виде, а именно:

$$
\Psi=\operatorname{Symm}\left\{\varphi_{1}\left(x_{1}\right) \varphi_{2}\left(x_{2}\right) \ldots \varphi_{N}\left(x_{N}\right)\right\}
$$

где Symm - симметризатор. В этом случае сушествует набор решений $\varphi_{i}(x)$ с "почти" перекрываюшимися носителями при $\hbar \rightarrow 0$, поэтому при $\hbar \rightarrow 0$ надо пренебречь обменными членами, которые экспоненциально малы по $\hbar$, и получится система уравнений Хартри точно такая же, как в фермионном случае. В действительности для математически строгой теории переход к системе уравнений Хартри совершается с помощью приведенного выше асимптотического приема (см. (1)-(3)), причем в левую и правую части уравнения (1) (cp. $[1,2,4])$ вставляется оператор симметризации, что не изменяет его симметрических решений.

Далее возникают задачи: 1) нахождения асимптотики других серий, а не только тех, которые получил Боголюбов; 2) нахождения асимптотики некоторых серий без условия малости взаимодействия (или плотности).

Известно, что для фермионов с помощью идеи куперовских пар была получена асимптотика некоторых серий лиш при условии $N \rightarrow \infty$ ( $N$ - число частиц) без условия малости взаимодействия. На самом деле с чисто математической точки зрения была получена асимптотика серии некоторого осредненного оператора Шредингера.

5. Пусть $k, M \geqslant 0$ - целые числа. Рассмотрим гильбертово пространство функций вида

$$
\Psi\left(x_{1}, y_{1} ; \ldots ; x_{M}, y_{M} ; z_{1}, \gamma_{1}, j_{1} ; \ldots ; z_{k}, \gamma_{k}, j_{k}\right)
$$

где $x_{l}, y_{l}, z_{m} \in \mathbf{T}^{3}, j_{m}=1, \ldots, \infty$ для всех $m=1, \ldots, k$ и $l=1, \ldots, M$, которые удовлетворяют условиям: а) функции симметричны относительно перестановок любых пар переменных $\left(x_{l}, y_{l}\right)$ и $\left(x_{m}, y_{m}\right)$, б) они симметричны относительно перестановок любых 
пар переменных $\left(z_{l}, \gamma_{l}, j_{l}\right)$ и $\left(z_{m}, \gamma_{m}, j_{m}\right)$. Скалярное произведение имеет вид

$$
\begin{aligned}
& \sum_{\gamma_{1}, \ldots, \gamma_{k} \in I} \sum_{j_{1}, \ldots, j_{k}=1}^{\infty} \int d x_{1} d y_{1} \ldots d x_{M} d y_{M} d z_{1} \ldots d z_{k} \times \\
& \quad \times \Psi^{*}\left(x_{1}, y_{1} ; \ldots ; x_{M}, y_{M} ; z_{1}, \gamma_{1}, j_{1} ; \ldots ; z_{k}, \gamma_{k}, j_{k}\right) \times \\
& \quad \times \Phi\left(x_{1}, y_{1} ; \ldots ; x_{M}, y_{M} ; z_{1}, \gamma_{1}, j_{1} ; \ldots ; z_{k}, \gamma_{k}, j_{k}\right) \stackrel{\text { def }}{=}(\Psi, \Phi) .
\end{aligned}
$$

Будем обозначать это пространство $\mathcal{F}_{M, k}$.

ОПРЕДЕЛЕНИЕ. ОПределим бозонное подпространство $\mathcal{F}_{M, k}^{B}$. Пространство $\mathcal{F}_{M, k}^{B}$ является подпространством пространства $\mathcal{F}_{M, k}$ и состоит из таких элементов $\mathcal{F}_{M, k}$, которые симметричны относительно перестановок любых $\left(x_{i}, \alpha_{i}\right)$ и $\left(z_{l}, \sigma_{l}\right)$ и симметричны относительно перестановок любых $j_{l}$ и $j_{m}$. Проектор на это подпространство будет обозначаться как $\widehat{P}_{M, k}^{B}$ :

$$
\widehat{P}_{M, k}^{B}: \mathcal{F}_{M, k} \rightarrow \mathcal{F}_{M, k}^{B}
$$

Рассмотрим теперь гильбертово пространство $\mathcal{F}$, элементами которого являются бесконечные наборы $\Psi=\left\{\Psi_{M, k}\right\}_{M, k}, \Psi_{M, k} \in \mathcal{F}_{M, k}$,

$$
\|\Psi\|^{2}=\sum_{M, k}\left\|\Psi_{M, k}\right\|^{2}<\infty .
$$

Это пространство является бесконечной прямой суммой пространств $\mathcal{F}=\bigoplus_{M, k} \mathcal{F}_{M, k}$ и называется пространством Фока [5]. Отметим, что пространство $\mathcal{F}$ отличается от обычных пространств Фока, используемых при описании многочастичных систем. Обозначим оператор проектирования на соответствуюшее слагаемое прямой суммы как $\widehat{P}_{M, k}$ :

$$
\widehat{P}_{M, k}: \mathcal{F} \rightarrow \mathcal{F}_{M, k}, \quad \widehat{P}_{M, k} \Psi=\Psi_{M, k},
$$

а оператор вложения $\mathcal{F}_{M, k}$ в $\mathcal{F}$ как $\hat{i}_{M, k}$ :

$$
\hat{i}_{M, k}: \mathcal{F}_{M, k} \rightarrow \mathcal{F}, \quad \hat{i}_{M, k} \varphi=\left\{\delta_{M m} \delta_{k s} \varphi\right\}_{m, s}
$$

Пусть задан набор операторов $\left\{\hat{A}_{N}\right\}_{N}$ в пространстве функций

$$
\psi_{N}\left(x_{1} ; \ldots ; x_{N}\right) \in L^{2}
$$

симметричных относительно перестановок переменных $\left(x_{i}\right)$ и $\left(x_{j}\right), x_{i} \in \mathbf{T}^{3}$. Им отвечают операторы $\tilde{\hat{A}}_{m, s}$ на подпространствах $\mathcal{F}_{m, s}^{B}$, действуюшие как единичные по дискретным переменным $j_{l}$. Осредненным оператором $\overline{\hat{A}}$ будем называть оператор в пространстве Фока $\mathcal{F}$ :

$$
\overline{\hat{A}}=\sum_{k=0}^{\infty} \sum_{m=0}^{\infty} \frac{(2 m+k) !}{4^{m} m !} \hat{i}_{m, k} \tilde{\hat{A}}_{2 m+k} \widehat{P}_{m, k}^{B} \widehat{P}_{m, k}
$$

6 Теоретическая и математическая физика, т. 121, № 3, 1999 г. 
Пример. Пусть $\hat{A}_{N}=\hat{\mathbf{E}}$, где $\hat{\mathbf{E}}$ - тождественный оператор. Осредненный оператор в бозонном случае имеет вид

$$
\overline{\hat{\mathbf{E}}}=\sum_{k=0}^{\infty} \sum_{m=0}^{\infty} \frac{(2 m+k) !}{4^{m} m !} \hat{i}_{m, k} \widehat{P}_{m, k}^{F} \widehat{P}_{m, k} .
$$

Пусть задан некоторый элемент $\Psi \in \mathcal{F}$. Обозначим

$$
\begin{aligned}
\bar{M} & =\frac{\sum_{k=0}^{\infty} \sum_{m=0}^{\infty} \frac{(2 m+k) !}{4^{m} m !} m\left(\Psi, \hat{i}_{m k} \widehat{P}_{m k}^{B} \widehat{P}_{m k} \Psi\right)}{\sum_{k=0}^{\infty} \sum_{m=0}^{\infty} \frac{(2 m+k) !}{4^{m} m !}\left(\Psi, \hat{i}_{m k} \widehat{P}_{m k}^{F} \widehat{P}_{m k} \Psi\right)} \\
\bar{k} & =\frac{\sum_{k=0}^{\infty} \sum_{m=0}^{\infty} \frac{(2 m+k) !}{4^{m} m !} k\left(\Psi, \hat{i}_{m k} \widehat{P}_{m k}^{B} \widehat{P}_{m k} \Psi\right)}{\sum_{k=0}^{\infty} \sum_{m=0}^{\infty} \frac{(2 m+k) !}{4^{m} m !}\left(\Psi, \hat{i}_{m k} \widehat{P}_{m k}^{F} \widehat{P}_{m k} \Psi\right)}
\end{aligned}
$$

ЗАмечАниЕ. Оператор $\hat{i}_{M, k} \widehat{H}_{k+2 M} \widehat{P}_{M, k}^{B} \widehat{P}_{M, k}$ может быть выражен следуюшим образом:

$$
\begin{aligned}
& \frac{1}{M ! k !} \sum_{j_{1}=1}^{\infty} \ldots \sum_{j_{k}=1}^{\infty} \int \ldots \int d x_{1} d y_{1} \ldots d x_{M} d y_{M} d z_{1} \ldots d z_{k} \times \\
& \quad \times \widehat{B}^{+}\left(x_{1}, y_{1}\right) \ldots \widehat{B}^{+}\left(x_{M}, y_{M}\right) \hat{b}^{+}\left(z_{1}, j_{1}\right) \ldots \hat{b}^{+}\left(z_{k}, j_{k}\right) \widehat{H}_{k+2 M} \times \\
& \quad \times \operatorname{Symm}_{x_{1}, y_{1}, \ldots, x_{M}, y_{M}, z_{1}, \ldots, z_{k}} \operatorname{Symm}_{j_{1}, \ldots, j_{k}}\left\{\widehat{B}^{-}\left(x_{M}, y_{M}\right) \ldots \widehat{B}^{-}\left(x_{1}, y_{1}\right) \times\right. \\
& \left.\quad \times \hat{b}^{-}\left(z_{1}, j_{1}\right) \ldots \hat{b}^{-}\left(z_{k}, j_{k}\right)\right\} \times \\
& \quad \times \exp \left(-\sum_{j=1}^{\infty} \int d z \hat{b}^{+}(z, j) \hat{b}^{-}(z, j)\right) \exp \left(-\iint d x d y \widehat{B}^{+}(x, y) \widehat{B}^{-}(x, y)\right) .
\end{aligned}
$$

6. Получим теперь уравнения нестандартных характеристик для многочастичной бозонной задачи. Для этого произведем переход во всех операторных выражениях (7) к $c$-числам. Для фиксированного числа бозонов $N$ при фиксированном $k$ - числе параметров $j_{1}, \ldots, j_{k}, j_{s}=1, \ldots, J$, от которых зависит волновая функция в уравнении Шредингера, - мы определяем гамильтониан, отвечающий $1 / N$-характеристикам [1] для уравнения (7), из следуюшего соотношения:

$$
\begin{aligned}
H_{k, M}[ & \left.\Phi^{*}(x, y), \Phi(x, y) ; \varphi^{*}(x, j), \varphi(x, j)\right] \sum_{j_{1}=1}^{\infty} \ldots \sum_{j_{k}=1}^{\infty} \int \ldots \int d x_{1} \ldots d x_{N} \times \\
& \times \varphi^{*}\left(x_{1}, j_{1}\right) \ldots \varphi^{*}\left(x_{k}, j_{k}\right) \Phi^{*}\left(x_{k+1}, x_{k+2}\right) \ldots \Phi^{*}\left(x_{k+2 M-1}, x_{k+2 M}\right) \times \\
& \times \operatorname{Symm}_{x_{1} \ldots x_{k+2 M}}\left\{\Phi\left(x_{k+1}, x_{k+2}\right) \ldots \Phi\left(x_{k+2 M-1}, x_{k+2 M}\right) \times\right. \\
& \left.\times \varphi\left(x_{1}, j_{1}\right) \ldots \varphi\left(x_{k}, j_{k}\right)\right\}= \\
= & \sum_{j_{1}=1}^{\infty} \ldots \sum_{j_{k}=1}^{\infty} \int \ldots \int d x_{1} \ldots d x_{N} \varphi^{*}\left(x_{1}, j_{1}\right) \ldots \varphi^{*}\left(x_{k}, j_{k}\right) \times
\end{aligned}
$$




$$
\begin{aligned}
& \times \Phi^{*}\left(x_{k+1}, x_{k+2}\right) \ldots \Phi^{*}\left(x_{k+2 M-1}, x_{k+2 M}\right) H_{k+2 M}(x) \times \\
& \times \operatorname{Symm}_{x_{1} \ldots x_{k+2 M}}\left\{\Phi\left(x_{k+1}, x_{k+2}\right) \ldots \Phi\left(x_{k+2 M-1}, x_{k+2 M}\right) \times\right. \\
& \left.\times \varphi\left(x_{1}, j_{1}\right) \ldots \varphi\left(x_{k}, j_{k}\right)\right\}
\end{aligned}
$$

где $M=(N-k) / 2$. Просуммируем эти уравнения при разных значениях $k$ и $M$, умножим при этом правую и левую части уравнения на коэффициент

$$
A_{k, M}=\frac{(k+2 M) !}{4^{M}(M !)^{2} k !} .
$$

Получим следуюшее уравнение для функции $H_{\text {mean }}\left[\Phi^{*}(x, y), \Phi(x, y) ; \varphi^{*}(x, j), \varphi(x, j)\right]$, которую будем называть усредненным гамильтонианом характеристик многочастичной бозонной задачи:

$$
\begin{aligned}
H_{\text {mean }}[ & \left.\Phi^{*}(x, y), \Phi(x, y) ; \varphi^{*}(x, j), \varphi(x, j)\right] \sum_{k=0}^{\infty} \sum_{M=0}^{\infty} \frac{(k+2 M) !}{4^{M}(M !)^{2} k !} \times \\
& \times \sum_{j_{1}=1}^{\infty} \ldots \sum_{j_{k}=1}^{\infty} \int \ldots \int d x_{1} \ldots d x_{k+2 M} \varphi^{*}\left(x_{1}, j_{1}\right) \ldots \varphi^{*}\left(x_{k}, j_{k}\right) \times \\
& \times \Phi^{*}\left(x_{k+1}, x_{k+2}\right) \ldots \Phi^{*}\left(x_{k+2 M-1}, x_{k+2 M}\right) \times \\
& \times \operatorname{Symm}_{x_{1} \ldots x_{k+2 M}}\left\{\Phi\left(x_{k+1}, x_{k+2}\right) \ldots \Phi\left(x_{k+2 M-1}, x_{k+2 M}\right) \times\right. \\
& \left.\times \varphi\left(x_{1}, j_{1}\right) \ldots \varphi\left(x_{k}, j_{k}\right)\right\}= \\
= & \sum_{k=1}^{\infty} \ldots \sum_{M=0}^{\infty} \frac{(k+2 M) !}{4^{M}(M !)^{2} k !} \sum_{j_{1}=1}^{\infty} \sum_{j_{k}=1}^{\infty} \int \cdots \int d x_{1} \ldots d x_{k+2 M} \varphi^{*}\left(x_{1}, j_{1}\right) \ldots \times \\
& \times \cdots \varphi^{*}\left(x_{k}, j_{k}\right) \Phi^{*}\left(x_{k+1}, x_{k+2}\right) \ldots \Phi^{*}\left(x_{k+2 M-1}, x_{k+2 M}\right) H_{k+2 M}(x) \times \\
& \times \operatorname{Symm}_{x_{1} \ldots x_{k+2 M}}\left\{\Phi\left(x_{k+1}, x_{k+2}\right) \ldots \Phi\left(x_{k+2 M-1}, x_{k+2 M}\right) \times\right. \\
& \left.\times \varphi\left(x_{1}, j_{1}\right) \ldots \varphi\left(x_{k}, j_{k}\right)\right\} .
\end{aligned}
$$

Используя представление вторичного квантования [5] для симметричных функций, представим уравнение (8) в виде

$$
\begin{aligned}
H_{\text {mean }} & {\left[\Phi^{*}(x, y), \Phi(x, y) ; \varphi^{*}(x, j), \varphi(x, j)\right] \times } \\
& \times\left(\Xi_{0}, \exp \left(\frac{1}{2} \iint d x d y \Phi^{*}(x, y) \stackrel{2}{\psi}^{-}(x) \stackrel{2}{\psi^{-}}(y)\right) \times\right. \\
& \times \exp \left(\sum_{j=1}^{\infty} \iint d z d w \varphi^{*}(z, j) \varphi(w, j) \stackrel{2}{\psi^{-}}(z) \stackrel{1}{\psi^{+}}(w)\right) \times \\
& \left.\times\left(\frac{1}{2} \iint d x^{\prime} d y^{\prime} \Phi\left(x^{\prime}, y^{\prime}\right) \stackrel{1}{\psi}^{+}\left(x^{\prime}\right) \stackrel{1}{\psi}^{+}\left(y^{\prime}\right)\right) \Xi_{0}\right)= \\
= & \left(\Xi_{0}, \exp \left(\frac{1}{2} \iint d x d y \Phi^{*}(x, y) \stackrel{3}{\psi}^{-}(x) \stackrel{3}{\psi}^{-}(y)\right) \times\right.
\end{aligned}
$$




$$
\begin{aligned}
& \times \exp \left(\sum_{j=1}^{\infty} \iint d z d w \varphi^{*}(z, j) \varphi(w, j) \stackrel{3}{\psi}^{-}(z) \stackrel{1}{\psi}+(w)\right) \stackrel{2}{\hat{H}}_{\mathrm{F}} \times \\
& \left.\times \exp \left(\frac{1}{2} \iint d x^{\prime} d y^{\prime} \Phi\left(x^{\prime}, y^{\prime}\right) \stackrel{1}{\psi^{+}}\left(x^{\prime}\right) \stackrel{1}{\psi^{+}}\left(y^{\prime}\right)\right) \Xi_{0}\right),
\end{aligned}
$$

где $\psi^{-}(x), \psi^{+}(x)$ - операторы в бозонном пространстве Фока [5], которые удовлетворяют коммутационным соотношениям

$$
\left[\psi^{-}(x), \psi^{+}(y)\right]=\delta(x-y), \quad\left[\psi^{ \pm}(x), \psi^{ \pm}(y)\right]=0,
$$

$\Xi_{0}$ - вакуумный вектор пространства Фока, т.е. элемент этого пространства, который удовлетворяет условиям

$$
\psi^{-}(x) \Xi_{0}=0, \quad\left(\Xi_{0}, \Xi_{0}\right)=1,
$$

$\widehat{H}_{\mathrm{F}}-$ оператор в пространстве Фока,

$$
\widehat{H}_{\mathrm{F}}=\int d x \psi^{+}(x)\left(-\frac{\hbar^{2}}{2 m} \Delta\right) \psi^{-}(x)+\frac{1}{2} \iint d x d y V(x-y) \psi^{+}(x) \psi^{+}(y) \psi^{-}(y) \psi^{-}(x),
$$

а число над оператором обозначает порядок его действия [4]. Аналогично формуле (8) определим среднее число "одиночных" бозонов $\bar{k}$ и среднее число пар бозонов $\bar{M}$ как функции гамильтоновых переменных $\Phi^{*}(x, y), \Phi(x, y), \varphi^{*}(x, j), \varphi(x, j)$ :

$$
\begin{aligned}
\bar{k} \sum_{k=0}^{\infty} & \sum_{M=0}^{\infty} \frac{(k+2 M) !}{4^{M}(M !)^{2} k !} \sum_{j_{1}=1}^{\infty} \ldots \sum_{j_{k}=1}^{\infty} \int \ldots \int d x_{1} \ldots d x_{k+2 M} \varphi^{*}\left(x_{1}, j_{1}\right) \ldots \varphi^{*}\left(x_{k}, j_{k}\right) \times \\
& \times \Phi^{*}\left(x_{k+1}, x_{k+2}\right) \ldots \Phi^{*}\left(x_{k+2 M-1}, x_{k+2 M}\right) \times \\
& \times \operatorname{Symm}_{x_{1} \ldots x_{k+2 M}}\left\{\Phi\left(x_{k+1}, x_{k+2}\right) \ldots \Phi\left(x_{k+2 M-1}, x_{k+2 M}\right) \times\right. \\
& \left.\times \varphi\left(x_{1}, j_{1}\right) \ldots \varphi\left(x_{k}, j_{k}\right)\right\}= \\
= & \sum_{k=1}^{\infty} \sum_{M=0}^{\infty} k \frac{(k+2 M) !}{4^{M}(M !)^{2} k !} \sum_{j_{1}=1}^{\infty} \ldots \sum_{j_{k}=1}^{\infty} \int \ldots \int d x_{1} \ldots d x_{k+2 M} \varphi^{*}\left(x_{1}, j_{1}\right) \ldots \times \\
& \times \ldots \varphi^{*}\left(x_{k}, j_{k}\right) \Phi^{*}\left(x_{k+1}, x_{k+2}\right) \ldots \Phi^{*}\left(x_{k+2 M-1}, x_{k+2 M}\right) \times \\
& \times \operatorname{Symm}_{x_{1} \ldots x_{k+2 M}}\left\{\Phi\left(x_{k+1}, x_{k+2}\right) \ldots \Phi\left(x_{k+2 M-1}, x_{k+2 M}\right) \times\right. \\
& \left.\times \varphi\left(x_{1}, j_{1}\right) \ldots \varphi\left(x_{k}, j_{k}\right)\right\}, \\
\bar{M} \sum_{k=0}^{\infty} & \sum_{M=0}^{\infty} \frac{(k+2 M) !}{4^{M}(M !)^{2} k !} \sum_{j_{1}=1}^{\infty} \ldots \sum_{j_{k}=1}^{\infty} \int \ldots \int d x_{1} \ldots d x_{k+2 M} \varphi^{*}\left(x_{1}, j_{1}\right) \ldots \varphi^{*}\left(x_{k}, j_{k}\right) \times \\
& \times \Phi^{*}\left(x_{k+1}, x_{k+2}\right) \ldots \Phi^{*}\left(x_{k+2 M-1}, x_{k+2 M}\right) \times \\
& \times \operatorname{Symm}_{x_{1} \ldots x_{k+2} M}\left\{\Phi\left(x_{k+1}, x_{k+2}\right) \ldots \Phi\left(x_{k+2 M-1}, x_{k+2 M}\right) \times\right. \\
& \left.\times \varphi\left(x_{1}, j_{1}\right) \ldots \varphi\left(x_{k}, j_{k}\right)\right\}= \\
= & \sum_{k=1}^{\infty} \sum_{M=0}^{\infty} \sum_{M} \frac{(k+2 M) !}{4{ }^{M}(M !)^{2} k !} \sum_{j_{1}=1}^{\infty} \ldots \sum_{j_{k}=1}^{\infty} \int \ldots \int d x_{1} \ldots d x_{k+2 M} \varphi^{*}\left(x_{1}, j_{1}\right) \ldots \times \\
&
\end{aligned}
$$




$$
\begin{aligned}
& \times \cdots \varphi^{*}\left(x_{k}, j_{k}\right) \Phi^{*}\left(x_{k+1}, x_{k+2}\right) \ldots \Phi^{*}\left(x_{k+2 M-1}, x_{k+2 M}\right) \times \\
& \times \operatorname{Symm}_{x_{1} \ldots x_{k+2 M}}\left\{\Phi\left(x_{k+1}, x_{k+2}\right) \ldots \Phi\left(x_{k+2 M-1}, x_{k+2 M}\right) \times\right. \\
& \left.\times \varphi\left(x_{1}, j_{1}\right) \ldots \varphi\left(x_{k}, j_{k}\right)\right\}
\end{aligned}
$$

Формулы (10), (11) с использованием представления вторичного квантования можно представить в виде

$$
\begin{aligned}
& \bar{k}=\frac{\partial}{\partial t} \ln \left(\left(\Xi_{0}, \exp \left(\frac{1}{2} \iint d x d y \Phi^{*}(x, y) \stackrel{2}{\psi^{-}}(x) \stackrel{2}{\psi^{-}}(y)\right) \times\right.\right. \\
& \times \exp \left(t \sum_{j=1}^{J} \iint d z d w \varphi^{*}(z, j) \varphi(w, j) \stackrel{2}{\psi^{-}}(z) \stackrel{1}{\psi^{+}}(w)\right) \times \\
& \left.\left.\times \exp \left(\frac{1}{2} \iint d x^{\prime} d y^{\prime} \Phi\left(x^{\prime}, y^{\prime}\right) \stackrel{1}{\psi^{+}}\left(x^{\prime}\right) \stackrel{1}{\psi^{+}}\left(y^{\prime}\right)\right) \Xi_{0}\right)\right)\left.\right|_{t=1} \\
& \bar{M}=\frac{\partial}{\partial t} \ln \left(\left(\Xi_{0}, \exp \left(\frac{\sqrt{t}}{2} \iint d x d y \Phi^{*}(x, y) \stackrel{2}{\psi^{-}}(x) \stackrel{2}{\psi^{-}}(y)\right) \times\right.\right. \\
& \times \exp \left(\sum_{j=1}^{J} \iint d z d w \varphi^{*}(z, j) \varphi(w, j) \stackrel{2}{\psi^{-}}(z) \stackrel{1}{\psi}+(w)\right) \times \\
& \left.\left.\times \exp \left(\frac{\sqrt{t}}{2} \iint d x^{\prime} d y^{\prime} \Phi\left(x^{\prime}, y^{\prime}\right) \stackrel{1}{\psi^{+}}\left(x^{\prime}\right) \stackrel{1}{\psi^{+}}\left(y^{\prime}\right)\right) \Xi_{0}\right)\right)\left.\right|_{t=1} .
\end{aligned}
$$

Так как уравнение (1) инвариантно относительно сдвига вдоль тора, то

$$
\Phi(x, y)=\frac{1}{L^{3}} \sum_{p} \phi_{p} e^{i p(y-x)}, \quad \varphi(x, j)=\frac{1}{L^{3 / 2}} \varphi_{j} e^{i q_{j} x} \text { при } j=1, \ldots, \infty
$$

где $p=\frac{2 \pi}{L}\left(n_{1}, n_{2}, n_{3}\right), n_{1}, n_{2}, n_{3}$ - целые числа, а $q_{j}$ является взаимнооднозначным отображением множества натуральных чисел на счетное множество векторов такого вида, что $\phi_{p}=\phi_{-p}$, а также введено обозначение

$$
\sum_{p}=\sum_{n_{1}=-\infty}^{\infty} \sum_{n_{2}=-\infty}^{\infty} \sum_{n_{3}=-\infty}^{\infty}
$$

Будем далее использовать обозначение $\varphi_{p}$ для $\varphi_{j(p)}$, где $j(p)$ - обратное отображение описанного вьше множества векторов на множество натуральных чисел. На функциях (14) гамильтониан имеет вид

$$
H_{\text {mean }}\left[\Phi^{*}(x, y), \Phi(x, y), \varphi^{*}(x, j), \varphi(x, j)\right]=\widetilde{H}\left[\phi_{p}^{*}, \phi_{p}, \varphi_{p}^{*}, \varphi_{p}\right]
$$


где $\widetilde{H}\left[\phi_{p}^{*}, \phi_{p}, \varphi_{p}^{*}, \varphi_{p}\right]$ определяется из соотношения

$$
\begin{aligned}
\widetilde{H}\left[\phi_{p}^{*}, \phi_{p}, \varphi_{p}^{*}, \varphi_{p}\right]\left(\Xi_{0}, \exp \left(\frac{1}{2} \sum_{p} \phi_{p}^{*} \psi_{p}^{-} \psi_{-p}^{-}\right) \exp \left(\sum_{q}\left|\varphi_{q}\right|^{2} \psi_{q}^{-}{ }^{1} \psi_{q}^{+}\right) \times\right. \\
\left.\quad \times \exp \left(\frac{1}{2} \sum_{r} \phi_{r} \psi_{r}^{+}{ }^{+} \psi_{-r}^{+}\right) \Xi_{0}\right)= \\
=\left(\Xi_{0}, \exp \left(\frac{1}{2} \sum_{p} \phi_{p}^{*} \dot{\psi}_{p}^{-} \psi_{-p}^{-}\right) \exp \left(\sum_{q}\left|\varphi_{q}\right|^{2}{ }^{3} \psi_{q}^{-}{ }^{-} \psi_{q}^{+}\right) \widehat{\widehat{H}}_{\mathrm{F}} \times\right. \\
\left.\quad \times \exp \left(\frac{1}{2} \sum_{r} \phi_{r} \psi_{r}^{+} \psi_{-r}^{+}\right) \Xi_{0}\right),
\end{aligned}
$$

где операторы $\psi_{p}^{ \pm}$имеют вид

$$
\psi_{p}^{-}=\frac{1}{L^{3 / 2}} \int d x \psi^{-}(x) e^{-i p x}, \quad \psi_{p}^{+}=\frac{1}{L^{3 / 2}} \int d x \psi^{+}(x) e^{i p x},
$$

причем для них выполняются соотношения

$$
\left[\psi_{p}^{-}, \psi_{r}^{+}\right]=\delta_{p r}, \quad\left[\psi_{p}^{ \pm}, \psi_{r}^{ \pm}\right]=0, \quad \psi_{p}^{-} \Xi_{0}=0 .
$$

Очевидно, что

$$
\widehat{H}_{\mathrm{F}}=\sum_{p} \frac{\hbar^{2} p^{2}}{2 m} \psi_{p}^{+} \psi_{p}^{-}+\frac{1}{2 L^{3}} \sum_{p} \sum_{r} \sum_{s} \widetilde{V}(s) \psi_{p+s}^{+} \psi_{r-s}^{+} \psi_{r}^{-} \psi_{p}^{-},
$$

где $\widetilde{V}(s)=\int d x V(x) e^{i s x}$. Среднее число одиночных бозонов и среднее число пар для функций (14) выражаются следующим образом:

$$
\begin{aligned}
\bar{k}= & \frac{\partial}{\partial t} \ln \left(\left(\Xi_{0}, \exp \left(\frac{1}{2} \sum_{p} \phi_{p}^{*} \psi_{p}^{-} \stackrel{2}{\psi}_{-p}^{-}\right) \exp \left(t \sum_{q}\left|\varphi_{q}\right|^{2} \dot{\psi}_{q}^{-}{ }^{1} \psi_{q}^{+}\right) \times\right.\right. \\
& \left.\left.\times \exp \left(\frac{1}{2} \sum_{r} \phi_{r} \stackrel{1}{\psi}_{r}^{+}{ }^{+} \psi_{-r}^{+}\right) \Xi_{0}\right)\right)\left.\right|_{t=1}, \\
\bar{M}= & \frac{\partial}{\partial t} \ln \left(\left(\Xi_{0}, \exp \left(\frac{\sqrt{t}}{2} \sum_{p} \phi_{p}^{*} \dot{\psi}_{p}^{-} \stackrel{2}{\psi}_{-p}^{-}\right) \exp \left(\sum_{q}\left|\varphi_{q}\right|^{2} \psi_{q}^{-}{ }^{-1} \psi_{q}^{+}\right) \times\right.\right. \\
& \left.\left.\times \exp \left(\frac{\sqrt{t}}{2} \sum_{r} \phi_{r} \psi_{r}^{+}{ }^{+} \psi_{-r}^{+}\right) \Xi_{0}\right)\right)\left.\right|_{t=1} .
\end{aligned}
$$

Упростим выражения (15), (17), (18). Для этого рассмотрим следуюший оператор:

$$
A_{p}(t)=\exp \left(t \psi_{p}^{-}{ }_{p}^{+}+\right)
$$


Этот оператор, очевидно, удовлетворяет уравнению

$$
\frac{\partial A_{p}}{\partial t}(t)=\psi_{p}^{-} A_{p}(t) \psi_{p}^{+}
$$

с начальным условием $A_{p}(0)=1$, где 1 обозначает единичный оператор. Воспользуемся следующим известным тождеством (для константы $c$ ):

$$
\stackrel{2}{\psi_{p}^{-}} \exp \left(c \psi_{p}^{+}\right)=(\stackrel{1}{\psi}-\bar{p}+c) \exp \left(c \psi_{p}^{+}\right),
$$

и вместо числа $c$ подставим оператор $\stackrel{3}{\psi} \underset{p}{-}$, действующий третьим [4]. Тогда уравнение (20) принимает вид

$$
\frac{\partial A_{p}}{\partial t}(t)=A_{p}(t) \psi_{p}^{-} \psi_{p}^{+}+t \psi_{p}^{-} A_{p}(t) \psi_{p}^{+}=A_{p}(t) \psi_{p}^{-} \psi_{p}^{+}+t \frac{\partial A_{p}}{\partial t}(t),
$$

откуда для оператора (19) получается уравнение

$$
\frac{\partial A_{p}}{\partial t}(t)=\frac{1}{1-t} A_{p}(t) \psi_{p}^{-} \psi_{p}^{+}
$$

решение которого с учетом начального условия имеет вид

$$
A_{p}(t)=\exp \left(-\ln (1-t) \psi_{p}^{-} \psi_{p}^{+}\right)=\exp \left(-\ln (1-t)\left(1+\psi_{p}^{+} \psi_{p}^{-}\right)\right) .
$$

Таким образом, оператор (19) при $|t|<1$ имеет вид (21). Рассмотрим также следующие элементы пространства Фока при $p \neq 0$ :

$$
\exp \left(t \psi_{-p}^{-} \psi_{p}^{-}\right) \exp \left(\theta_{0} \psi_{p}^{+} \psi_{-p}^{+}\right) \Xi_{0}
$$

При $|t|<1 / \theta_{0}$ имеет место равенство

$$
\exp \left(t \psi_{-p}^{-} \psi_{p}^{-}\right) \exp \left(\theta_{0} \psi_{p}^{+} \psi_{-p}^{+}\right) \Xi_{0}=\frac{1}{1-t \theta_{0}} \exp \left(\frac{\theta_{0}}{1-t \theta_{0}} \psi_{p}^{+} \psi_{-p}^{+}\right) \Xi_{0},
$$

которое доказывается аналогично формулам (19)-(21). В случае, когда $p=0$, получим, что при $|t|<1 /\left(4 \theta_{0}\right)$ справедливо соотношение

$$
\exp \left(t \psi_{0}^{-} \psi_{0}^{-}\right) \exp \left(\theta_{0} \psi_{0}^{+} \psi_{0}^{+}\right) \Xi_{0}=\frac{1}{\sqrt{1-4 t \theta_{0}}} \exp \left(\frac{\theta_{0}}{1-4 t \theta_{0}} \psi_{0}^{+} \psi_{0}^{+}\right) \Xi_{0} .
$$

Из равенств (21), (23), (24) следует, что

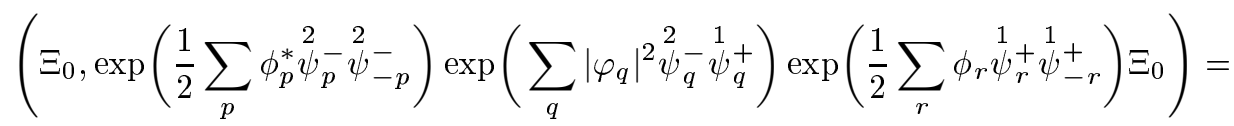

$$
\begin{aligned}
& =\left(\Xi_{0}, \exp \left(\frac{1}{2} \sum_{p} \phi_{p}^{*} \psi_{p}^{-} \psi_{-p}^{-}\right) \prod_{q} \exp \left(-\ln \left(1-\left|\varphi_{q}\right|^{2}\right)\left(1+\psi_{q}^{+} \psi_{q}^{-}\right)\right) \times\right. \\
& \left.\times \exp \left(\frac{1}{2} \sum_{r} \phi_{r} \psi_{r}^{+} \psi_{-r}^{+}\right) \Xi_{0}\right)=\prod_{p} \frac{1}{\sqrt{\left(1-\left|\varphi_{p}\right|^{2}\right)\left(1-\left|\varphi_{-p}\right|^{2}\right)-\phi_{p}^{*} \phi_{p}}} .
\end{aligned}
$$


Используя (25), получим выражения для среднего числа одиночных бозонов (17) и среднего числа пар (18):

$$
\begin{aligned}
\bar{k} & =\sum_{p} \frac{\varphi_{p}^{*} \varphi_{p}\left(1-\varphi_{-p}^{*} \varphi_{-p}\right)}{\left(1-\varphi_{p}^{*} \varphi_{p}\right)\left(1-\varphi_{-p}^{*} \varphi_{-p}\right)-\phi_{p}^{*} \phi_{p}}, \\
\bar{M} & =\frac{1}{2} \sum_{p} \frac{\phi_{p}^{*} \phi_{p}}{\left(1-\varphi_{p}^{*} \varphi_{p}\right)\left(1-\varphi_{-p}^{*} \varphi_{-p}\right)-\phi_{p}^{*} \phi_{p}} .
\end{aligned}
$$

Имеют место следующие очевидные тождества:

$$
\begin{aligned}
& \frac{\partial}{\partial\left|\varphi_{s}\right|^{2}}\left(\Xi_{0}, \exp \left(\frac{1}{2} \sum_{p} \phi_{p}^{*} \psi_{p}^{-}{ }^{2}{ }_{-p}^{-}\right) \exp \left(\sum_{q}\left|\varphi_{q}\right|^{2}{ }^{2}{ }_{q}^{-}{ }^{1} \psi_{q}^{+}\right) \exp \left(\frac{1}{2} \sum_{r} \phi_{r}{ }_{r}^{+}{ }_{r}^{+} \psi_{-r}^{+}\right) \Xi_{0}\right)= \\
& =\left(\Xi_{0}, \exp \left(\frac{1}{2} \sum_{p} \phi_{p}^{*} \stackrel{2}{p}_{p}^{-}{ }^{2}{ }_{-p}^{-}\right) \times\right. \\
& \left.\times \exp \left(\sum_{q}\left|\varphi_{q}\right|^{2} \psi_{q}^{-} \psi_{q}^{+}\right) \stackrel{2}{\psi}_{s}^{-} \psi_{s}^{+} \exp \left(\frac{1}{2} \sum_{r} \phi_{r}{ }^{1} \psi_{r}^{+} \psi_{-r}^{+}\right) \Xi_{0}\right), \\
& \frac{\partial^{2}}{\partial\left|\varphi_{s}\right|^{2} \partial\left|\varphi_{t}\right|^{2}}\left(\Xi_{0}, \exp \left(\frac{1}{2} \sum_{p} \phi_{p}^{*} \psi_{p}^{-}{ }^{2} \psi_{-p}^{-}\right) \exp \left(\sum_{q}\left|\varphi_{q}\right|^{2} \psi_{q}^{-} \psi_{q}^{+}\right) \times\right. \\
& \left.\times \exp \left(\frac{1}{2} \sum_{r} \phi_{r} \stackrel{1}{\psi}_{r}^{+} \stackrel{1}{\psi}_{-r}^{+}\right) \Xi_{0}\right)= \\
& =\left(\Xi_{0}, \exp \left(\frac{1}{2} \sum_{p} \phi_{p}^{*} \stackrel{2}{\psi}_{p}^{-} \psi_{-p}^{-}\right) \exp \left(\sum_{q}\left|\varphi_{q}\right|^{2}{ }^{2} \psi_{q}^{-}{ }^{+} \psi_{q}^{+}\right) \stackrel{2}{\psi_{s}^{-}}{ }_{s}^{1} \psi_{s}^{+} \times\right. \\
& \left.\times \stackrel{2}{\psi_{t}^{-}} \psi_{t}^{+} \exp \left(\frac{1}{2} \sum_{r} \phi_{r}{ }_{r}^{+}{ }_{r}^{+} \psi_{-r}^{+}\right) \Xi_{0}\right) \\
& 4 \frac{\partial^{2}}{\partial \phi_{s}^{*} \partial \phi_{t}}\left(\Xi_{0}, \exp \left(\frac{1}{2} \sum_{p} \phi_{p}^{*} \psi_{p}^{-} \psi_{-p}^{-}\right) \exp \left(\sum_{q}\left|\varphi_{q}\right|^{2} \psi_{q}^{-} \psi_{q}^{+}\right) \times\right. \\
& \left.\times \exp \left(\frac{1}{2} \sum_{r} \phi_{r}{ }^{1} \psi_{r}^{+}{ }^{+}{ }_{-r}^{+}\right) \Xi_{0}\right)= \\
& =\left(\Xi_{0}, \exp \left(\frac{1}{2} \sum_{p} \phi_{p}^{*} \psi_{p}^{-} \stackrel{2}{\psi}_{-p}^{-}\right) \stackrel{2}{\psi_{s}^{-}} \stackrel{2}{\psi}_{-s}^{-} \exp \left(\sum_{q}\left|\varphi_{q}\right|^{2}{ }^{2} \psi_{q}^{-} \stackrel{1}{\psi_{q}^{+}}\right) \stackrel{1}{\psi}_{t}^{+}{ }_{t}^{1} \psi_{-t}^{+} \times\right. \\
& \left.\times \exp \left(\frac{1}{2} \sum_{r} \phi_{r} \psi_{r}^{+}{ }^{1} \psi_{-r}^{+}\right) \Xi_{0}\right) .
\end{aligned}
$$

Используя тождества (28), (29) и формулу (25), получим для гамильтониана 
$\widetilde{H}\left[\phi_{p}^{*}, \phi_{p}, \varphi_{p}^{*}, \varphi_{p}\right](15)$ следуюшее выражение:

$$
\begin{aligned}
\widetilde{H}\left[\phi_{p}^{*}, \phi_{p}, \varphi_{p}^{*}, \varphi_{p}\right]= & \sum_{p} \frac{\hbar^{2} p^{2}}{2 m} G_{p}+\frac{1}{2 L^{3}} \sum_{p} \sum_{q}(\widetilde{V}(0)+\widetilde{V}(p-q)) G_{p} G_{q}+ \\
& +\frac{1}{2 L^{3}} \sum_{p} \sum_{q} \widetilde{V}(p-q) R_{p}^{*} R_{q},
\end{aligned}
$$

где $G_{p}, R_{p}$ имеют вид

$$
\begin{aligned}
G_{p} & =\frac{\left|\varphi_{p}\right|^{2}}{1-\left|\varphi_{p}\right|^{2}}+\frac{1}{1-\left|\varphi_{p}\right|^{2}} \frac{\phi_{p}^{*} \phi_{p}}{\left(1-\left|\varphi_{p}\right|^{2}\right)\left(1-\left|\varphi_{-p}\right|^{2}\right)-\phi_{p}^{*} \phi_{p}}, \\
R_{p} & =\frac{\phi_{p}}{\left(1-\left|\varphi_{p}\right|^{2}\right)\left(1-\left|\varphi_{-p}\right|^{2}\right)-\phi_{p}^{*} \phi_{p}} .
\end{aligned}
$$

Очевидно, что $G_{p}$ являются действительными. Из формул (31)-(33) следует, что гамильтоновы переменные $\varphi_{p}^{*}, \varphi_{p}$ входят в усредненный гамильтониан только в комбинациях $\varphi_{p}^{*} \varphi_{p}$. Из этого вытекает, что гамильтониан $\widetilde{H}\left[\phi_{p}^{*}, \phi_{p}, \varphi_{p}^{*}, \varphi_{p}\right]$ имеет бесконечный набор интегралов движения $\varphi_{p}^{*} \varphi_{p}$. Зафиксируем значения этих интегралов движения, а также среднее число одиночных бозонов $\bar{k}$ и среднее число пар $\bar{M}$; кроме того, пусть $\varphi_{p}=\varphi_{-p}$. После выделения циклических переменных гамильтониана $\widetilde{H}\left[\phi_{p}^{*}, \phi_{p}, \varphi_{p}^{*}, \varphi_{p}\right]$ может быть найдено множество точек покоя. Каждой точке покоя отвечает квазипериодическая траектория усредненного гамильтониана характеристик $H_{\text {mean }}\left[\Phi^{*}(x, y)\right.$, $\left.\Phi(x, y) ; \varphi^{*}(x, j), \varphi(x, j)\right]$. Из формул $(25),(28),(29),(31)-(33)$ следует уравнение для $\phi_{p}^{*}, \phi_{p}$ :

$$
\begin{aligned}
\left(\widetilde{\varepsilon}_{p}\right. & -\lambda) R_{p}+\frac{1}{2 L^{3}} \sum_{q} \widetilde{V}(p-q) R_{q}\left(1+G_{p}\right)+\frac{1}{2 L^{3}} \sum_{q} \widetilde{V}(p-q) R_{q}^{*} \frac{R^{2}}{\left(1+G_{p}\right)}= \\
& =(\mu-\lambda) \frac{R_{p}\left(1+G_{p}\right)}{\left(1+G_{p}\right)^{2}-R_{p}^{*} R_{p}} .
\end{aligned}
$$

Здесь $\lambda$ и $\mu$ - две частоты, определяюшие траекторию, а $\widetilde{\varepsilon}_{p}$ имеют вид

$$
\widetilde{\varepsilon}_{p}=\frac{\hbar^{2} p^{2}}{2 m}+\frac{1}{L^{3}} \sum_{q}(\widetilde{V}(0)+\widetilde{V}(p-q)) G_{q} .
$$

Поскольку $\operatorname{Im} G_{p}=0$, то из уравнения (34) следует уравнение

$$
\frac{1}{L^{3}} \sum_{q} \widetilde{V}(p-q) R_{q} R_{p}^{*}=\frac{1}{L^{3}} \sum_{q} \widetilde{V}(p-q) R_{q}^{*} R_{p} .
$$

В силу (36) уравнение (34) приводится к виду

$$
\begin{aligned}
\left(\widetilde{\varepsilon}_{p}-\mu\right) R_{p}+\frac{1}{2 L^{3}} \sum_{q} \tilde{V}(p-q) R_{q}\left(1+2 G_{p}\right)= \\
=\frac{1}{2 L^{3}} \sum_{q} \tilde{V}(p-q) R_{q} \frac{G_{p}\left(1+G_{p}\right)-R_{p}^{*} R_{p}}{\left(1+G_{p}\right)}- \\
\quad-(\mu-\lambda) R_{p} \frac{G_{p}\left(1+G_{p}\right)-R_{p}^{*} R_{p}}{\left(1+G_{p}\right)^{2}-R_{p}^{*} R_{p}} .
\end{aligned}
$$


Система уравнений (36), (37) может быть представлена в виде

$$
\left[A_{p}, L_{p}\right]=0
$$

где операторы $A_{p}, L_{p}$ являются матрицами $2 \times 2$ :

$$
\begin{aligned}
L_{p} & =\left(\begin{array}{cc}
\widetilde{\varepsilon}_{p}-\mu & -\Delta_{p}^{*} \\
\Delta_{p} & -\widetilde{\varepsilon}_{p}+\mu
\end{array}\right), \\
A_{p} & =\left(\begin{array}{cc}
G_{p}+\frac{1}{2} & R_{p}^{*} \\
-R_{p} & -G_{p}-\frac{1}{2}
\end{array}\right),
\end{aligned}
$$

где

$$
\begin{aligned}
\Delta_{p}= & \frac{1}{L^{3}} \sum_{q} \tilde{V}(p-q) R_{q}-\frac{1}{L^{3}} \sum_{q} \tilde{V}(p-q) R_{q} \frac{G_{p}\left(1+G_{p}\right)-R_{p}^{*} R_{p}}{\left(1+G_{p}\right)\left(1+2 G_{p}\right)}+ \\
& +2(\mu-\lambda) \frac{R_{p}}{1+2 G_{p}} \frac{G_{p}\left(1+G_{p}\right)-R_{p}^{*} R_{p}}{\left(1+G_{p}\right)^{2}-R_{p}^{*} R_{p}} .
\end{aligned}
$$

Для произвольной функции $f(\xi)$ выражение $A_{p}=f\left(L_{p}\right)$ удовлетворяет $(38)$. В силу "пуантилистического" постулата [6] полагаем

$$
f(\xi)=\frac{1}{e^{\xi / \Theta}-1}+\frac{1}{2},
$$

$\Theta$ - температура. В этом случае получим уравнения для $G_{p}, R_{p}$ :

$$
G_{p}=\frac{f\left(\lambda_{p}\right)}{\lambda_{p}} \widetilde{\varepsilon}_{p}-\frac{1}{2}, \quad R_{p}=-\frac{f\left(\lambda_{p}\right)}{\lambda_{p}} \Delta_{p}
$$

где $\lambda_{p}=\sqrt{\left(\widetilde{\varepsilon}_{p}-\mu\right)^{2}-\left|\Delta_{p}\right|^{2}}$. Константы $\lambda$ и $\mu$ функционально связаны со средним числом одиночных бозонов и средним числом пар. Отсюда следует, что при температуре, равной нулю, число одиночных бозонов не равно нулю. По аналогии с фермионным случаем

$$
\lambda_{p}=\sqrt{\left(\widetilde{\varepsilon}_{p}-\mu\right)^{2}-\left|\Delta_{p}\right|^{2}}
$$

дают спектр коллективных возбуждений в бозонной системе.

7. Условие действительности $\lambda_{p}$ затрудняет переход к классическому пределу при $\hbar \rightarrow 0$. Однако если имеется внешнее медленно меняющееся поле, то такой переход возможен. В этом случае скорость также является термодинамической величиной и переход через величину критической скорости звука можно считать фазовым переходом.

Сушественную роль здесь играет осреднение операторов, произведенное выше. В классике такое осреднение проводится для $N$-частичного оператора Лиувилля:

$$
\begin{aligned}
& \widehat{\mathcal{L}}_{N} \psi_{N}\left(q_{1}, p_{1}, \sigma_{1} ; \ldots ; q_{N}, p_{N}, \sigma_{N}\right) \equiv \\
& \quad \equiv-i\left(\sum_{j=1}^{N} p_{j} \frac{\partial}{\partial q_{j}}+\sum_{1 \leqslant j<l \leqslant N} \frac{\partial V\left(q_{j}-q_{l}\right)}{\partial q_{j}}\left(\frac{\partial}{\partial p_{l}}-\frac{\partial}{\partial p_{j}}\right)\right) \psi_{N}=\gamma \psi_{N}
\end{aligned}
$$


где $\psi_{N} \in L^{2}$ - антисимметрическая (в случае фермионов) или симметрическая (в случае бозонов) функция относительно перестановок переменных $\left(q_{j}, p_{j}, \sigma_{j}\right)$ и $\left(q_{k}, p_{k}, \sigma_{k}\right)$ при $j, k=1, \ldots, N$ и где $q_{j}, p_{j} \in \mathbf{T}^{3}, \sigma_{j}$ - спиновая переменная, пробегаюшая конечное множество значений.

При рассмотрении классических фермионов, так же как при рассмотрении квантовых фермионов, уравнение для одиночных фермионов удовлетворяет уравнению для пар и уравнения для $R$ и $G$ не зависят от того, сколько одиночных фермионов имеется в исходной задаче. Этот удивительный факт имеет место только для фермионов. Поэтому можно предполагать, что именно пары играют ключевую роль в фазовом переходе для фермионов. В случае бозонов это свойство не имеет места. Значит, возможно, тройки, четверки и т.д. бозонов играют не меньшую роль, чем пары. А возможно, и достаточно большие кластеры из бозонов играют ключевую роль в фазовых переходах для симметрических решений уравнения Лиувилля. Отметим между прочим, что в физической литературе рассматриваются всегда именно симметрические решения уравнения Лиувилля для многих частищ, т.е. по нашей “вольной" терминологии классические бозоны.

Суммирование для обшего случая выглядит следуюшим образом:

$$
\bar{A}=\frac{\sum_{k_{1}=0}^{N} \cdots \sum_{k_{N}=0}^{N} \frac{\left(\sum_{i=1}^{N} i k_{i}\right) !}{\prod_{i=1}^{N}\left(k_{i} !\right)^{i-1}} \prod_{j=1}^{N} \varepsilon_{j}^{k_{j}}\left(\Psi, \hat{A} \hat{i}_{k_{1}, \ldots, k_{N}} \widehat{P}_{k_{1}, \ldots, k_{N}}^{B} \widehat{P}_{k_{1}, \ldots, k_{N}} \Psi\right)}{\sum_{k_{1}=0}^{N} \cdots \sum_{k_{N}=0}^{N} \frac{\left(\sum_{i=1}^{N} i k_{i}\right) !}{\prod_{i=1}^{N}\left(k_{i} !\right)^{i-1}} \prod_{j=1}^{N} \varepsilon_{j}^{k_{j}}\left(\Psi, \hat{i}_{k_{1}, \ldots, k_{N}} \widehat{P}_{k_{1}, \ldots, k_{N}}^{B} \widehat{P}_{k_{1}, \ldots, k_{N}} \Psi\right)},
$$

где $N=\sum_{i=1}^{N} i k_{i}$ - среднее число бозонов, $k_{i}$ - число кластеров, содержаших $i$ бозонов, операторы $\hat{i}_{k_{1}, \ldots, k_{N}}, \widehat{P}_{k_{1}, \ldots, k_{N}}^{B}, \widehat{P}_{k_{1}, \ldots, k_{N}}$ определяются по аналогии с введенными выше операторами $\hat{i}_{k_{1}, k_{2}}, \widehat{P}_{k_{1}, k_{2}}^{B}$ и $\widehat{P}_{k_{1}, k_{2}}$. Это суммирование даже для пар достаточно сложно, и мы не имеем возможности здесь его привести.

Разумеется, спектр уравнения Лиувилля для симметрических решений содержит асимптотику разности собственных значений не только бозонных решений уравнения Шредингера. Однако скачок термодинамических величин для чисто бозонных квантовых решений, если он сохраняется при $\hbar \rightarrow 0$, не может не сказаться на скачке аналогичных термодинамических величин для уравнения Лиувилля. Таких скачков для “классических бозонов" может быть только больше, т.е. могут появиться лишние, “паразитные" фазовые переходы.

8. Фазовый переход по скорости заманчиво трактовать как переход в состояние, вызывающее турбулентное течение - макроскопическую осредненную по объему $V$ хаотизацию. В сверхтекучей жидкости хаотическое движение дает принципиально другую функцию распределения [6], чем указанная выше статистическая сумма $\Sigma$, вычисленная на основании (5), (6). Так, безусловно, переход к осредненному хаотическому движению можно было бы рассматривать как фазовый.

Хотя эта трактовка для классической жидкости достаточно неожиданна и даже парадоксальна, имеется ряд аналогий с обычными фазовыми переходами:

1. Скачок плотности компоненты, отвечающей движению со скоростью $v_{0}$, приводит в силу известного соотношения Бачинского [7] к скачку вязкости. Отметим, что Прандтль рассматривал для турбулентных течений те же уравнения Навье-Стокса, но с турбулентной вязкостью. 
2. Ситуация, когда с помощью сглаживания шероховатостей на поверхности трубы и устранения других возмушений добиваются увеличения критического числа Рейнольдса, идентична ситуации метастабильных состояний, например в случае переохлажденной жидкости. И в том, и в другом случае достаточно малейшего возмушения и наступает “фазовый переход” в лед или турбулентность.

3. Уленбек пишет [8], что если производить эксперименты по рассеянию света вблизи точек потери устойчивости, то можно заметить большое сходство с поведением вблизи критической точки перехода газ-жидкость. Это означает, что флуктуационная картина вблизи точки “фазового перехода” также идентична (см. $[9,10])$.

Заметим в заключение следуюшее. Фазовые переходы связаны с некоторыми особенностями статистической суммы. Точная статистическая сумма вида

$$
\sum=\sum_{n} \exp \left(\frac{-\lambda_{n}}{\Theta}\right),
$$

где $\lambda_{n}$ - набор положительных возрастаюших не слишком медленно по $n$ чисел, всегда аналитична по $\Theta$ при $\Theta>0$, так что в точной статсумме как бы и нет фазовых переходов по температуре. Фазовые переходы и негладкость возникают лишь в главном члене асимптотики $\sum$ по каким-либо малым параметрам. Эти параметры в нашем случае $\frac{1}{N}$ ( $N$ - число частиц $)$ и константа Планка $\hbar$, которые находятся в некотором сочетании со временем релаксации $t_{0}$. Если число частиц, например, недостаточно велико или время менњше времени релаксации, то фазовый переход размывается. Значит, когда мы говорим о фазовом переходе, мы пренебрегаем величиной этой переходной зоны.

Если принять гипотезу о фазовом переходе ламинарного потока, увлеченного трубой в турбулентное течение, то надо пренебречь зоной перехода от ламинарного течения к полностью турбулентному течению. Только если эту зону и время релаксации считать сравнительно малыми, то тогда лишш можно говорить о переходе в турбулентное течение как о фазовом переходе.

Благодарности. Автор приносит глубокую благодарность Г. В. Ковалю за помощь при проведении вычислений и И. А. Фомину за исключительно полезные дискуссии.

\section{Список литературы}

[1] В. П. Маслов. Теория возмушений и асимптотические методы. М.: МГУ, 1965.

[2] В. П. Маслов. Комплексный метод ВКБ в нелинейных уравнениях. М.: Наука, 1977.

[3] V. P. Maslov. Russian J. Math. Phys. 1995. V. 2. № 4. P. 527-534; V. 3. № 1. P. 123-132.

[4] В. П. Маслов. Операторные методы. М.: Наука, 1973.

[5] Ф. А. Березин. Метод вторичного квантования. М.: Наука, 1965.

[6] V. P. Maslov. Russian J. Math. Phys. 1996. V. 5. № 4. P. 478-488.

[7] A. Batchinski. Z. Phys. Chem. 1913. V. 84. P. 643.

[8] Г. Уленбек. УФН. 1971. Т. 103. № 2. С. 75-318.

[9] П. Гленсдор $\phi$ И. Пригожин. Термодинамическая теория структуры, устойчивости и флуктуаций. М.: Мир, 1973.

[10] В. В. Струминский. О возможности применения динамических методов для описания турбулентных течений. Турбулентные течения. М.: Наука, 1974.

Поступила в редакцию 13.IV.1998 г., после доработки 20.IX.1999 г. 